\title{
Increasing Antimicrobial Resistance Monitored in Surveillance Analysis of Blood Stream Infections in Febrile Neutropenic Pediatric Oncology Patients
}

\author{
Hadir A El-Mahallawy ${ }^{1}$, Safaa Shawky Hassan¹, Mohamed El-Wakil ${ }^{2 *}$, Manar \\ M Moneer ${ }^{3}$, Lobna Shalaby ${ }^{4}$
}

\begin{abstract}
Background: Continuous surveillance of pattern of blood stream infection is necessary in febrile neutropenia (FN)especially with the recent escalating trend in the management of pediatric cancer patients towards intensified regimens and with the increase in infections caused by resistant organisms limiting the choice of antibiotics. Aim: To monitor change in pattern of blood stream infections (BSI) in FN pediatric cancer patients. Materials and Methods: Surveillance of FN episodes with positive BSI was prospectively monitored and compared to a previous surveillance in the same pediatric oncology unit. Results: A total of 232 BSI positive episodes were documented in 192 patients during a 6 months period. The results of recent surveillance analysis showed an increase in intensified regimens of chemotherapy, antimicrobial resistance, fungal infections, and prolonged duration of episodes when compared to previous surveillance, with $p$ value sof $<0.001,0.005,0.021$, and $<0.001$, respectively. There was an apparent decrease in the crude mortality but this was not statistically significant, to 6\% in 2011 from $10 \%$ in 2006. Conclusions: The pattern of BSI at our institution is still inclining towards gram positive organisms but is showing a shift towards more antibiotic resistance and fungal infections.
\end{abstract}

Keywords: Blood stream infections (BSI) - Febrile neutropenia (FN) - multidrug resistant organism (MDRO)

Asian Pac J Cancer Prev, 16 (14), 5691-5695

\section{Introduction}

Blood stream infections are significant causes of morbidity and mortality in hematology and oncology patients (Rodriguez et al., 2012; Sancho et al., 2012). Duration and intensity of neutropenia are important risk factors for bacteremia and poor outcomes in patients with cancer (Samonis et al., 2013). Hematology patients and hematopoietic stem cell transplant (HSCT) recipients undergoing intensive myelosuppressive or immunosuppressive treatment are at high risk for severe, life-threatening, bacterial infections. Thirteen to $60 \%$ of HSCT recipients develop BSI, which are associated with 12 to $42 \%$ mortality (Trecarichi et al., 2009). Monitoring local epidemiology is important in directing the strategy of management of febrile neutropenia. Based on the new evidence, international guidelines on antimicrobial prophylaxis have been recently updated, and targeted prophylaxis schedules have been proposed for different clinical settings (Corrado et al., 2011).

Growing resistance to standard antibiotics leads to increased use of broad-spectrum regimens, including carbapenems and combinations, with consequent collateral damage, including the selection of carbapenem- and multidrug resistant pathogens, predisposition to fungal infections and Clostridium difficile-associated diarrhea (EL-Mahallawy et al., 2011; Averbuch et al., 2013), Building recommendations for empirical therapy in this era of growing resistance is challenging (Giamarellou et al., 2010). Therefore, we attempted in this study to monitor the changing epidemiology that might have occurred in the pattern of blood stream infections in febrile neutropenic pediatric oncology patients in our institution.

\section{Materials and Methods}

The study was composed of 2 parts. A prospective part including surveillance of pediatric cases with cancer receiving chemotherapy and developing bacteremia while febrile neutopenic in the period from fist of April to the end of September 2011 at the Pediatric Oncology Department of National Institute (NCI), Cairo University, Egypt. The other part was comparing the 2011 surveillance to a previous one recorded in 2006 at the same unit (ELMahallawy et al., 2011). Patients who received a marrow transplant were not included in both cohorts. The study

${ }^{1}$ Clinical Pathology Department, ${ }^{3}$ Biostatistics and Cancer Epidemiology Department, ${ }^{4}$ Pediatric Oncology Department, National Cancer Institute, Cairo University Cairo, ${ }^{2}$ Clinical Oncology Department, Faculty of Medicine, Beni Suef University, Beni Suef, Egypt*For correspondence: wakilmohamed171@gmail.com 
was approved by NCI Ethics Committee and an informed consent was obtained from parents of participants.

Febrile neutropenia is defined as an oral temperature $>38.5^{\circ} \mathrm{C}$ or two consecutive readings of $>38.0^{\circ} \mathrm{C}$ for $2 \mathrm{~h}$ and an absolute neutrophil count $<0.5 \times 10^{9} / \mathrm{L}$, or expected to fall below $0.5 \times 10^{9} / \mathrm{L}$ (Naurois et al., 2010).

Data collected at first day of fever included age, diagnosis, state of disease, absolute neutrophil count (ANC), clinically documented infections and evidence of fungal infections, previous febrile episodes treated with broad spectrum antibiotics within one month preceding the current episode. Other data included were type of chemotherapy either standardized or intensified protocols. Intensified protocols are the regimens used in induction therapy, while standardized protocols are those used for maintenance. Follow up of patients was carried out as regard antibiotic received, need of shift, duration of episode and outcome of patients one month following febrile neutropenic episode.

\section{Microbiology}

Two blood cultures sets were usually drawn from each patient within the first day of fever, one from peripheral veins, and the other from cannula site, portacath, or CVC. Bactec 9050 incubator was used. Blood culture sampling was the same used in the study performed in 2006 (EL-Mahallawy et al., 2011). Identification of isolates was carried out using Microscan dried gram negative MIC/Combo and dried gram positive MIC/ Combo panels Seimens Healthcare Diagnostics Ltd (Sir William Siemens Sq. Frimley, Cambeley, UK GU16 8QD) for Gram-negative and Gram-positive organisms, respectively. The panel of antibiotics used for Gram negative included amikacin, amoxicillin-clavulanate, ampicillin-sulbactam, aztreonam, cefepime, cefoperazone, ceftazidime, cefuroxime, ciprofloxacin, gentamycin, imipenem, meropenem, norfloxacin, piperacillin, piperacillin-tazobactam, ticarcicilline-clavulanate and trimethoprim-sulfamethoxazole. Clindamycin, erythromycin, gatifloxacin, gentamycin, levofloxacin, linezolid, moxifloxacin, oxacillin, synercid, tetracycline, and vancomycin were the main antibiotics included in gram positive panels. Interpretation of susceptibility test results was done by referring to the CLSI guidelines. (Clinical and Laboratory Standards Institute. Performance standards for antimicrobial susceptibility testing. Nineteenth informational supplement M100-S19. Wayne, PA: Clinical and Laboratory Standards Institute; 2009). A multidrug resistant organism (MDRO) was defined as acquired non-susceptibility to at least one agent in three or more antimicrobial categories(Magiorakos et al., 2012); which was the same definition used in the evaluation of the 2006 patient cohort.

\section{Management}

During the febrile episode patients were hospitalized and treated with double agent broad spectrum antimicrobial therapy. The empirical regimen of hospital guidelines were cefepime/amikacine (FEP/AK), or piperacillintazobactem/amikacin (TZP/AK); with second line meropenem/amikacin (MEM/AK). Empirical antifungal was used if fever persisted till day 5. An antiviral (zovirax) was used when indicated. Antibiotic continued until patient became afebrile and ANC exceeded $0.5 \times 10^{9} \mathrm{~L}$. The episode was considered successfully controlled when fever and clinical signs resolved within 5-7 days. A prolonged episode was defined by persistent fever for more than 7 days, with other measures taken into consideration including the general condition of the patient as regards severity and duration of neutropenia, in addition to any associated clinical focus. Invasive fungal infections (IFI) were defined according to the criteria defined by the European Organization for Research and Treatment of Cancer (EORTC) (Ascioglu et al., 2002).

Statistical Methods Data were analyzed using SPSSwin statistical package version 15. Chi-square or Fisher's Exact tests were used to compare qualitative variables. Comparison between two groups regarding numeric variables was done using either Student's t-test or Mann-Whitney test as appropriate. Multivariate analysis was done using binary logistic regression by forward conditional method. $\mathrm{P}<0.05$ was considered significant. All tests were two tailed.

\section{Results}

\section{Patients Characteristic}

During 6 months period of 2011, 232 febrile bacteremic episodes were recorded in 192 patients. The patient age ranged from $1 \frac{1}{2}$ month to 18 years with a mean age of $6.8 \pm 4.5$. They were 119 males and 73 females. Their diagnoses were acute lymphoblastic leukemia, acute myeloblastic leukemia, lymphoma, and solid tumors in $72(37.5 \%), 36(18.7 \%), 21(11 \%)$, and 63 (32.8\%), respectively.

\section{Clinical Features}

The mean value of fever at day 1 was $38.5 \pm 0.5^{\circ} \mathrm{C}$. Clinical documented infections (CDI) were recorded in $114(49 \%)$ episodes, whereas, $118(51 \%)$ episodes lacked a focus. The sites of CDI were lower respiratory tract, diarrhea, or skin and intra-venous line related infections in $55(24 \%), 24(10 \%)$, and $14(6 \%)$, respectively. Other sites of infections included otitis media, tonsillitis, sinusitis, ophthalmic infection, herpetic lesions and chicken pox were reported in 68 (29\%) episodes. Previous febrile episodes with intake of empirical broad spectrum antibiotics were recorded in $142(61 \%)$ episode, while 90 (39\%) showed no previous febrile episode. Neutropenia was below $0.5 \times 10^{9} / \mathrm{L}$ in 163 episode $(70 \%)$ and extended for $\geq 7$ days in $122(53 \%)$ of episodes.

\section{Microbiology}

Gram-positive organisms (GPC) were the predominant causative agents of BSI, constituting 168 (72\%) of isolated organisms, while $56(24 \%)$ of BSIs were caused by Gram-negative organisms (GNR), and 8 (3\%) were mixed Infections. The organisms isolated are summarized in Table 1. Of the isolated pathogens, 119 (51\%) were MDRO. Comparison of percentage of multiresistance among some of the isolated organisms is illustrated in Figure 1. 
Invasive fungal infections was recorded in 53/232 (23\%) of episodes. Prolonged duration of neutropenia was significantly associated with IFI, as $79 \%(n=42)$ of cases

Table 1. Isolated Organisms from 232 Bacteremic Febrile Episodes in Pediatric Cancer Patients in 2011

\begin{tabular}{lcc}
\hline Organisms & Number $(\%)$ \\
\hline Gram-positive organisms & $168(72.4)$ \\
Coagulase negative Staphylococci & 135 & $(58.2)$ \\
Streptococcus spp. & 16 & $(6.9)$ \\
& $(7$ enterococci) \\
Stapylococcus aureus & $13 \quad(5.6)$ \\
& $(12$ MRSA) \\
Micrococci & 1 & $(0.4)$ \\
Mixed gram positive cocci & 3 & $(1.3)$ \\
Gram-negative organisms & 56 & $(24.1)$ \\
Non-fermenters & & \\
Acinetobacter sp. & 6 & $(2.6)$ \\
Pseudomonas sp. & 6 & $(2.6)$ \\
Stenotrophomonas maltophilia & 1 & $(0.4)$ \\
Enterobacteriacae & & \\
E. coli & 11 & $(4.7)$ \\
Klebsiella spp. & 16 & $(6.9)$ \\
Enterobacter spp. & 9 & $(3.9)$ \\
Salmonella spp. & 1 & $(0.4)$ \\
Citrobacter freundii & 1 & $(0.4)$ \\
Achromobacter sp & 3 & $(1.3)$ \\
Other GNR & & \\
Alcaligenis & 2 & $(0.9)$ \\
Mixed growth & 8 & $(3.4)$ \\
\hline
\end{tabular}

Table 2. Comparison between some Parameters Reported in Surveillance Analysis of Blood Stream Infections in Febrile Neutropenic Pediatric Patients with Cancer in $2011(\mathrm{~N}=192)$ and that done on 2006 ( $\mathrm{N}=193)$

\begin{tabular}{|c|c|c|c|}
\hline CParameter & $\begin{array}{c}2006 \\
\mathrm{~N}=239 \\
\mathrm{~N}(\%)\end{array}$ & $\begin{array}{c}2011 \\
N=232 \\
N(\%)\end{array}$ & p-value \\
\hline Age $($ mean \pm SD $)$ & $6.7 \pm 4.8$ & $6.8 \pm 4.5$ & 0.832 \\
\hline Day 7evaluation & & & $<0.001$ \\
\hline Fever & $103(43)$ & $145(62)$ & \\
\hline Recovery & $136(57)$ & $87(38)$ & \\
\hline Chemotherapy & & & $<0.001$ \\
\hline Standard & $129(54)$ & $74(32)$ & \\
\hline Intensified & $110(46)$ & $158(68)$ & \\
\hline Clinical infections & & & 0.492 \\
\hline No & $114(48)$ & $118(51)$ & \\
\hline Yes & $125(52)$ & $114(49)$ & \\
\hline Organism & & & 0.010 \\
\hline $\begin{array}{l}\text { Gram positive cocci } \\
\text { Gram negative rods }\end{array}$ & $180(75)$ & $168(72)$ & \\
\hline Mixed & $59(25)$ & $56(24)$ & \\
\hline $0(0)$ & $8(4)$ & & \\
\hline $\begin{array}{l}\text { Antibiotic resistance } \\
\text { Sensitive }\end{array}$ & & & 0.005 \\
\hline Resistant & $147(62)$ & 113 (49) & \\
\hline $92(38)$ & $119(51)$ & & \\
\hline Fungi & & & 0.021 \\
\hline No & $204(85.4 \%)$ & $179(77)$ & \\
\hline Yes & $35(14.6 \%)$ & $53(23)$ & \\
\hline Outcome & & & 0.120 \\
\hline Alive & $214(90)$ & $217(94)$ & \\
\hline Dead & $25(10)$ & $15(6)$ & \\
\hline
\end{tabular}

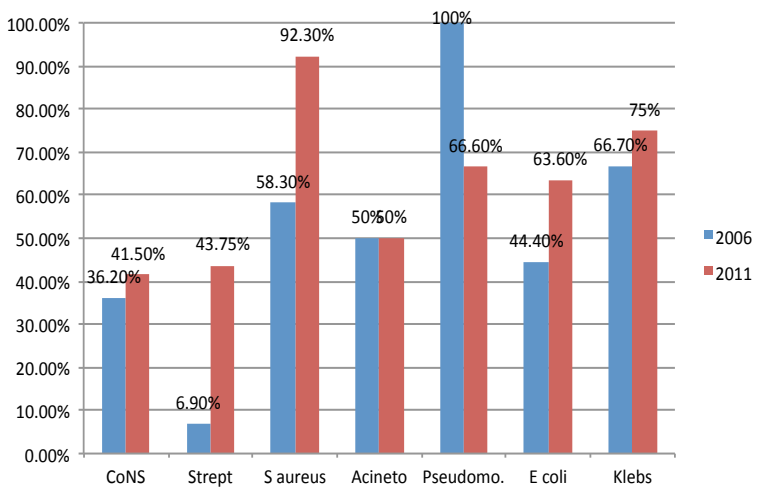

Figure 1. Comparison of Percentage of Multiresistance Among Isolated Organisms. CoNS, (Coagulase Negative Staphylococci), Strept (Streptococcal sp), S aureus (Staphylococcus Aureus), Acineto (Acinetobacter sp), Ps Aerug (Pseudomonas Aeruginosa), E. Coli (Escherechia Coli) Kleb (Klebsiella sp) between the two Periods in 2006\&2011

with evidence of IFI were showing $\geq 7$ days of neutropenia, versus $45 \%(n=81)$ of those with no IFI, in contrast to shorter duration of neutropenia in $21 \%(\mathrm{n}=11)$ of patients with IFI and $55 \%(n=98)$ of those with no IFI, $p<0.001$.

\section{Course and Outcome}

Recovery within 7 days was documented in $87(38 \%)$ of the episodes, whereas the infectious episode extended for 7 days or more in $145(62 \%)$ of the cases. The need to shift primary line of antibiotic was recorded in 55 (24\%) episode, while vancomycin was added in 120 (52\%) episodes. An empirical antifungal was given in $126(54 \%)$ episode. Continued use of antifungals after 7 days were recorded in 104 episodes (45\%). Crude mortality, i.e. mortality occurring within one month of infection was recorded in $15 / 232(6.5 \%)$ patients; $(8 / 168(4.8 \%)$ of the GPC, $12.5 \%, 7 / 56(12.5 \%)$ of the GNR).

The results of comparison of analysis of surveillance of blood stream infections (BSI) between over 6 months duration in 2006 and 2011 are demonstrated in Table 2.

\section{Discussion}

With the increased use of intensified chemotherapy in the growing population of patients with malignant disorders, profound and prolonged neutropenia are expected to occur with the resultant increase in the incidence of infections accompanied by difficulties in their management. Therefore, we attempted to investigate the change of epidemiology in FN pediatric cancer patients receiving chemotherapy at National Cancer Institute, its effect on morbidity and mortality, and the effectiveness of supportive care as regards need of antibiotic shift, duration and outcome of infectious episodes.

Pattern of blood stream infections in febrile neutropenia has passed through several shifts through the previous decades necessitating continuous surveillance over cancer centers worldwide to detect changes in local epidemiology and modify management guidelines accordingly. In the current study, we compared between surveillance results of 
BSI in pediatric cancer patients during a six months period in the year 2006 \& six months in the year 2011; GPC constituted $75 \%$ of organisms isolated in the year 2006 and $72 \%$ in 2011 . Whereas, gram negative pathogens were isolated in $25 \%$ and $24 \%$ of children with BSI in the years 2006 and 2011; respectively. In our institution, the pattern of BSI in pediatric cancer patients was characterized by predominance of gram positive bacteremia in several previous studies. (EL-Mahallawy et al., 2005; ELMahallawy et al., 2011). In recent years, the etiology of BSI in neutropenic patients in some cancer centers has shifted from gram-positive to gram-negative organisms (Gudiol et al., 2013), but this is in adult population. It is evident that pediatric patients are still having predominance of GPC as causes of BSIs. In the present study, there was an observed variation in the relative frequency of organisms within total GNRs with a decrease in the non-fermenters from $61 \%(36 / 59)$ in 2006 to $23 \%$ $(13 / 56)$ in 2011 and an increase in Enterobacteriacae in $2011(77 \%)$ compared to $2006(39 \%)$. This finding might be due to dedication of a specified infection control team concerned with applying strict infection control measures in the pediatric unit.

Though there was no change in the pattern of pathogens causing BSIs in pediatric cancer patients in our institution, a significant increase in antimicrobial resistance was recorded. There was an increased incidence of resistance in 2011 compared to 2006 as MDRO were significantly higher in $2011(51 \%)$ than in 2006 $(38.5 \%)$ ( $\mathrm{p}$ value 0.005$)$. There is a growing problem of antimicrobial resistance among the pathogens isolated from hematology patients and hematopoietic stem cell transplant recipients in many centers, and this increasingly influences the choice of empirical therapy (Averbuch et al., 2013). Previous intake of antibiotics in febrile neutropenic patients was reported to be the most frequent risk factor for BSI caused by MDROs (EL-Mahallawy et al., 2011; Mar et al., 2014). The increase of resistance recorded in the present study could be explained by that patients with hematological malignancies present with repeated febrile episodes and thus, receive repeated cycles of empirical antibiotics. Treatment options are very limited with increasing resistance, and the emergence of multiresistant Gram-negative organisms is inviting the use of old antibiotics, notably colistin/polymyxin B and fosfomycin2-6 and tigecycline (Livermore DM et al., 2014). Similarly, the emergence of Gram-positive pathogens with reduced susceptibility to glycopeptides is leading to the use of linezolid, daptomycin and tigecycline in hematology patients (Averbuch et al., 2013). Thus, modification of management guidelines is recommended in the era of growing resistance possibly by adopting a deescalation strategy to avoid the collateral damage initiated by antibiotic overuse (Averbuch et al., 2013).

Going on with the recent advances in the management of hematological malignancies towards intensified chemotherapy, more intensified regimens were used in the pediatric oncology department of NCI in the year 2011 (68\%), compared to the year $2006(46 \%) ; \mathrm{p}=0.001$. This is expected to be reflected on indicators of neutropenia, as intensified protocols will be significantly associated with more profound and prolonged duration of neutropenia. The increased use of intensified regimens of chemotherapy is accompanied by longer episodes and longer duration of neutropenia. The increased use of intensified regimens of chemotherapy seems to be accompanied by longer episodes and longer duration of neutropenia. Thus, it is evident that neutropenia is still a major determinant indicator of risk in febrile neutropenia.

Fungal infections are a growing problem in critically ill patients and are associated with increased morbidity and mortality (Paramythiotou et al., 2014). A significant increase in the incidence of IFI was recorded in the surveillance analysis of $2011(23 \%)$ when compared to that of $2006(15 \%)$; p value (0.021). The duration of neutropenia was a significant risk factor for the occurrence of IFI in the present study, p-value $<0.001$. Among the important risk factors of IFI described in many studies are those related to treatment of oncology cases, including prolonged ICU stay, treatment with corticosteroids, diabetes mellitus, central venous catheter, gastrointestinal surgery, total parenteral nutrition, prolonged antimicrobial use, immunosuppressive agents, chemotherapy, neutropenia, malnutrition, and multiple site colonization (Hoffmann-Santos et al., 2013). Thus, it is evident that the nowadays use of more intensified chemotherapy with its consequences on duration of neutropenia has increased the incidence of IFI necessitating clinical suspicion based on the awareness of its increasing frequency, and knowledge of local epidemiology for early diagnosis and prompt treatment of these potentially lethal opportunistic pathogens (Paramythiotou et al., 2014).

Despite advances in supportive care following cancer chemotherapy in recent practice, infections remain a major cause of morbidity, healthcare resource use and decline in efficacy as a result of delays in chemotherapy (Naurois $\mathbf{J}$ et al., 2010). In the present study, an unfavorable outcome was encountered in $6 \%(15 / 232)$ of the surveillance analysis of 2011 compared to $10 \%$ in 2006 . There is an apparent drop in mortality rate though it was not statistically different, yet the results are keeping on with the decreasing figures of mortality encountered for F\&N worldwide. Overall mortality rates are around 5\% in patients with solid tumors, as high as $11 \%$ in hematological malignancies, and is even higher in patients with proven bacteremia. The highest mortality is reported to be associated with GNRs, approaching a rate of $18 \%$ and $5 \%$ in GP bacteremia (Naurois et al., 2010).

Thus, it could be concluded from the comparison of results of febrile neutropenia surveillance in one cancer center five years apart that the pattern of BSI at our institution is still inclining towards gram positive organisms but is showing a big shift towards more antibiotic resistance. The increase in frequency of fungal infection in our patients could be due to the increased use of intensified chemotherapeutic protocols. This increase in frequency of IFI necessitate a change in the management approach towards prevention and prophylaxis in high risk groups rather than waiting for infection to occur. An apparent decline in figures of mortality in our institution invite us to follow up closely the pattern of FN in pediatric cancer patients for better performance and continuous 
progression in the field of supportive care.

This study is done as part of work of NCI and is funded by NCI.

No conflict of interest is considered for any of the authors of this study.

\section{References}

Ascioglu S, Rex JH, de Pauw W, et al (2002). Defining opportunistic invasive fungal infections in immunocompromised patients with cancer and hematopoietic stem cell transplants: an international consensus. Clin Infect Dis, 34, 7-14.

Averbuch D, Orasch C, Cordonnier C, et al (2013). European guidelines for empirical antibacterial therapy for febrile neutropenic patients in the era of emerging resistance: summary of the 4th European conference on infections in leukemia. Haematologica, 98, 1826-35.

Averbuch D, Cordonnier C, Livermore DM, et al (2013). Targeted therapy against multi-resistant bacteria in leukemic and hematopoietic stem cell transplant recipients: guidelines of the $4^{\text {th }}$ European conference on infections in leukemia (ECIL-4, 2011). Haematologica, 98, 1836-47.

Clinical and Laboratory Standards Institute (2009). Performance standards for antimicrobial susceptibility testing. Nineteenth informational supplement M100-S19. Wayne, PA: Clinical and Laboratory Standards Institute.

Corrado G, Francesco (2011). Current epidemiology and prevention of infectious complications in cancer patients. Euro Oncol Haematol, 7, 270-7.

El-Mahallawy HA, Sidhom I, El-Din NH, Zamzam M, El-Lamie MM (2005). Clinical and microbiological determinants of serious blood stream infections in Egyptian pediatric cancer patients : a one year study Int J Infect Dis, 9, 43-51

El-Mahallawy HA, El-Wakil M, Moneer MM, Shalaby L(2011). Antibiotic resistance is associated with longer bacteremic episodes and worse outcome in febrile neutropenic children with cancer. Pediatric Blood Cancer, 57, 238-88.

Giamarellou H (2010). Multidrug-resistant gram-negative bacteria: how to treat and for how long. Int $J$ Antimicrob Agents, 36, 50-4.

Gudiol C, Bodro M, Simonetti A, et al (2013). Changing aetiology, clinical features, antimicrobial resistance, and outcomes of bloodstream infection in neutropenic cancer patients. Clin Microbiol Infect, 19, 474-9.

Hoffmann-Santos HD, Paula CR, Yamamoto AC, Tadano T, Hahn RC (2013).Six-year trend analysis of nosocomial candidemia and risk factors in two intensive care hospitals in Mato Grosso, Midwest Region of Brazil. Mycopathologia, 176, 409-15.

Livermore DM, Warner M, Mushtaq S (2011). What remains against carbapenem-resistant Enterobacteriaceae? Evaluation of chloramphenicol, ciprofloxacin, colistin, fosfomycin, minocycline, nitrofurantoin, temocillin and tigecycline. Int $J$ Antimicrob Agents, 37, 415-9.

Magiorakos AP, Srinivasan A, Carey RB, et al (2012). Multidrugresistant, extensively drug-resistant and pandrug-resistant bacteria: an international expert proposal for interim standard definitions for acquired resistance. Clin Microbiol Infect, 18, 268-81.

Mar M, Carlota G, Carol G-V, Carmen A, Jordi C (2014). Bloodstream infections in patients with solid tumors: Epidemiology, antibiotic therapy, and outcomes in 528 episodes in a single center. Medicine, 93, 143-9.

Naurois J, Novitzky I, Basso, et al (2010). On behalf of the ESMO Guidelines Working Group. Management of febrile neutropenia: ESMO. Clin Practice GuidelinesAnn Oncol,
21, 252-6.

Paramythiotou, Frantzeska F, Flevari A, Armaganidis A, George DE (2014). Invasive fungal infections in the ICU: how to approach, how to treat. Molecules, 19, 1085-119.

Rodri guez L, Ethier MC, Phillips B, et al (2012). Utility of peripheral blood cultures in patients with cancer and suspected blood stream infections: A systematic review. Support Care Cancer, 20, 3261-7.

Sancho S, Artero A, Zaragoza R, et al (2012). Impact of nosocomial polymicrobial bloodstream infections on the outcome in critically ill patients. Eur J Clin Microbiol Infect Dis, 31, 1791-6.

Samonis G, Vardakas KZ, Maraki S, et al (2013). A prospective study of characteristics and outcomes of bacteremia in patients with solid organ or hematologic malignancies. Support Care Cancer, 21, 2521.

Trecarichi EM, Tumbarello M, Spanu T, et al (2009). Incidence and clinical impact of extended-spectrum-beta-lactamase (ESBL) production and fluoroquinolone resistance in bloodstream infections caused by Escherichia coli in patients with hematological malignancies. J Infect, 58, 299-307. 